\title{
3D Hollow Sn@Carbon-Graphene Hybrid Material as Promising Anode for Lithium-Ion Batteries
}

\author{
Xiaoyu Zheng, ${ }^{1}$ Wei Lv, ${ }^{2}$ Yan-Bing He, ${ }^{2}$ Chen Zhang, ${ }^{1}$ Wei Wei, ${ }^{1}$ \\ Ying Tao, ${ }^{1}$ Baohua Li, ${ }^{2}$ and Quan-Hong Yang ${ }^{1,2}$ \\ ${ }^{1}$ School of Chemical Engineering and Technology, Tianjin University, Tianjin 300072, China \\ ${ }^{2}$ Engineering Laboratory for Functionalized Carbon Materials, Graduate School at Shenzhen, Tsinghua University, \\ Shenzhen 518055, China \\ Correspondence should be addressed to Wei Lv; lv.wei@sz.tsinghua.edu.cn
}

Received 13 December 2013; Accepted 23 December 2013; Published 12 January 2014

Academic Editor: Zheng-Hong Huang

Copyright (C) 2014 Xiaoyu Zheng et al. This is an open access article distributed under the Creative Commons Attribution License, which permits unrestricted use, distribution, and reproduction in any medium, provided the original work is properly cited.

\begin{abstract}
A 3D hollow Sn@C-graphene hybrid material (HSCG) with high capacity and excellent cyclic and rate performance is fabricated by a one-pot assembly method. Due to the fast electron and ion transfer as well as the efficient carbon buffer structure, the hybrid material is promising in high-performance lithium-ion battery.
\end{abstract}

\section{Introduction}

Metallic Sn has long been considered as a promising anode material for lithium-ion batteries (LIBs) due to its high theoretical specific capacity $[1,2]$. However, $\mathrm{Sn}$ is plagued with a rapid capacity fading because of volume expansion induced pulverization during the lithiation and delithiation reactions, leading to the breakdown of electrical connection of anode particles [3, 4]. Generally, reducing the particle size to nanoscale is an effective way to minimize the volume change $[5,6]$. In order to further decrease the volume change of $\mathrm{Sn}$ in the electrochemical reactions, hollow $\mathrm{Sn}$ nanospheres (NSs), whose tensile stress is $\sim 5$ times lower than that of $\mathrm{Sn}$ solid NSs with an equal volume, are proven to be a better choice in electrochemical reactions [7-10].

Besides aforementioned routes, many researches also indicate that dispersing Sn NSs into carbon matrix is another effective approach to improve and stabilize the cyclability, where the carbon matrix restricts the volume expansion of Sn and moreover acts as an electron conductor to increase the conductivity [11-14]. A Sn core/carbon shell nanostructure is a typical structure to combine the advantages of carbon and Sn from above consideration, in which the carbon coating layers not only enhance the conductivity of electrode and buffer the volume variation but also help form a stable solid electrolyte interface (SEI) film.

Aggregation, which hinders the fast $\mathrm{Li}^{+}$transportation, is another problem for nanomaterials in real applications. Many latest results have shown that graphene nanosheets (GNs) are ideal substrates to well disperse NSs, which can also construct a flexible network through a "plane-to-point" mode to bridge the active material particles and form effective ion and electron transfer networks [15-18]. Furthermore, it is expected that such soft carbon layer could endure the volume change of the metal NSs and reduce the mechanical stress within the electrode to prevent its disintegration $[10,19$, 20].

Herein, we integrate the above concerns into one hybrid structure, and a three-dimensional hollow Sn@carbongraphene hybrid structure (abbreviated as HSCG) is obtained. In such an HSCG, hollow Sn NS was encapsulated in a carbon shell to form a core-shell sphere structure (hollow Sn@C) and these NSs are uniformly anchored onto the flexible GNs. On one hand, hollow Sn NSs are formed to decrease the intrinsic volume variation, and the carbon shell acts as a buffer layer to restrict the volume changes of Sn NS, which also improves the conductivity and helps the formation of stable SEI film. On the other hand, GNs are used as the substrate 


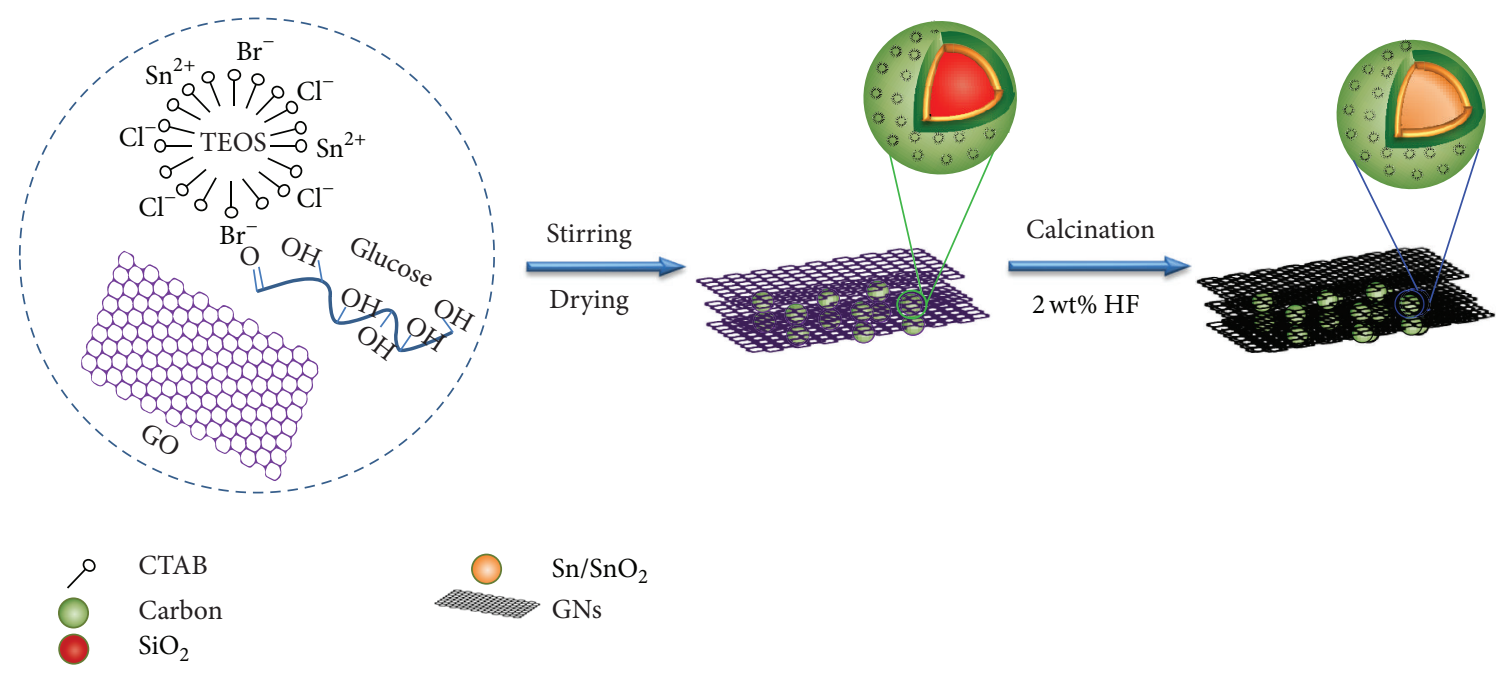

FIGURE 1: Scheme for the assembly process of hollow Sn@C-graphene hybrid nanostructure (HSCG).

to fix and isolate the NSs to avoid the aggregation and the presence of NSs prevents the restacking of GNs, which ensure the open ion transportation channels retention. The interlacing of GNs in the hybrid forms an interconnected conducting network, and the NSs are in a good contact with the GNs, which guarantee the electron transfer between the two components and the fabricated electrode. Furthermore, the GNs endure the volume change of the NSs at some extent. Therefore, the hybrid structure has combined the three above discussed characters, defined as fast ion transfer, interconnected conducting network, and efficient volume variation control in electrochemical performance improvement, and is a promising anode candidate for high performance LIBs [21, 22].

\section{Experimental}

2.1. Preparation of 3D Hollow Sn@C-Graphene. In a typical preparation process, $20 \mathrm{~mL}$ ammonia (26 wt\%) was added into $20 \mathrm{vol} \%$ ethanol aqueous solution, and $2.4 \mathrm{~g}$ hexadecyltrimethylammonium bromide (CTAB) was then dissolved into the above solution with continuous stirring for $20 \mathrm{~min}$. After that, $10 \mathrm{~mL}$ ethanol and $10 \mathrm{~mL}$ tetraethyl orthosilicate (TEOS) containing $5 \mathrm{~g} \mathrm{SnCl}_{2}$ were dropwise introduced into the above solution under stirring for $48 \mathrm{~h}$ and the obtained mixture solution was denoted as Mixture A. Mixture B was obtained by sonicating $1 \mathrm{~g}$ glucose and $400 \mathrm{mg}$ graphene oxide (GO) in $400 \mathrm{~mL}$ DI water with a probe sonicator (JY92-N, China, $300 \mathrm{~W}$ ) for $1 \mathrm{~h}$. Mixtures $\mathrm{A}$ and $\mathrm{B}$ were mixed under continuous stirring for $5 \mathrm{~h}$ followed by an overnight storage at room temperature. After dried at $120^{\circ} \mathrm{C}$, the obtained mixture was calcined at $800^{\circ} \mathrm{C}$ for $4 \mathrm{~h}$ under $\mathrm{N}_{2}$ and then treated with $\mathrm{HF}$ followed by washing with ethanol and DI water. Finally, the HSCG was obtained after dried at $120^{\circ} \mathrm{C}$ (Figure 1).

2.2. Structure Characterization. XRD measurements were conducted at room temperature using a specular reflection mode (Bruker D-8, Cu K $\alpha$ radiation, $\lambda=0.154056 \mathrm{~nm}$ ). SEM and TEM observations were performed by using Hitachi S-4800 (Hitachi, Japan) and JEM 2100F (JEOL, Japan), respectively. Nitrogen cryoadsorption was measured by using BEL mini-instrument, and the specific surface area was obtained by Brunauer-Emmett-Teller analyses of the adsorption isotherm. Elemental mapping was performed using energy-dispersive X-ray spectroscopy (EDX) in conjunction with a TEM. Thermogravimetric analysis (TGA) was carried out using TG 8120 (Thermo Plus) with a heating rate of $10^{\circ} \mathrm{C} / \mathrm{min}$ in a range of $25-900^{\circ} \mathrm{C}$ under air flow.

2.3. Electrochemical Performance Measurement. First, the asprepared sample as active material was ground into fine powders. $80 \mathrm{wt} \%$ active materials, $10 \mathrm{wt} \%$ Super P, and $10 \mathrm{wt} \%$ PTFE were well mixed in ethanol solution to make uniform mixture slurry under sonication. Then, the mixture slurry was spread uniformly on nickel foam and dried at $120^{\circ} \mathrm{C}$ for $12 \mathrm{~h}$ under vacuum to obtain a loading density of active material in the range of $2.2-2.5 \mathrm{mg} \mathrm{cm}^{-2}$. Finally, coin cells (CR2032) were assembled in Ar filled glove box and the lithium foils were used as the anode, and $1 \mathrm{M} \mathrm{LiPF}_{6}$ mixture solution $(1: 1(\mathrm{v} / \mathrm{v})$ of ethylene carbonate (EC) and dimethyl carbonate $(\mathrm{DMC}))$ as the electrolyte.

The coin cells were tested at room temperature using battery tester (Lixing, China) and electrochemistry workstation (Gamry Instrument, USA). Note that the charge and discharge processes were conducted at the same current density with the voltage range of $0.006 \mathrm{~V}-2.5 \mathrm{~V}$.

\section{Results and Discussion}

$\mathrm{X}$-ray diffraction (XRD) analysis was performed to indicate the reduction process from $\mathrm{Sn}^{2+}$ to metallic $\mathrm{Sn}$. As shown in Figure 2(a), the identified diffraction peaks that are located at $30.7^{\circ}, 32.1^{\circ}$, and $44.9^{\circ}$ are attributed to the tetragonal Sn (200), (101), and (211) planes, respectively (JCPDS Card no. 04-0673). Obviously, the initial $\mathrm{Sn}^{2+}$ was reduced into crystalline $\mathrm{Sn}$ by carbon at high temperature. According to the energy-dispersive X-ray (EDX) analysis, the residual $\mathrm{SiO}_{2}$ weight fraction is lower than $3 \%$, indicating that $\mathrm{SiO}_{2}$ has 


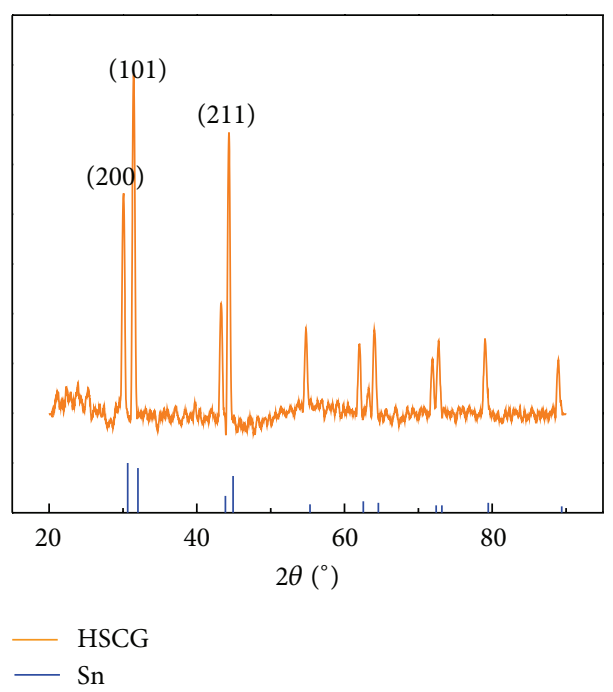

(a)

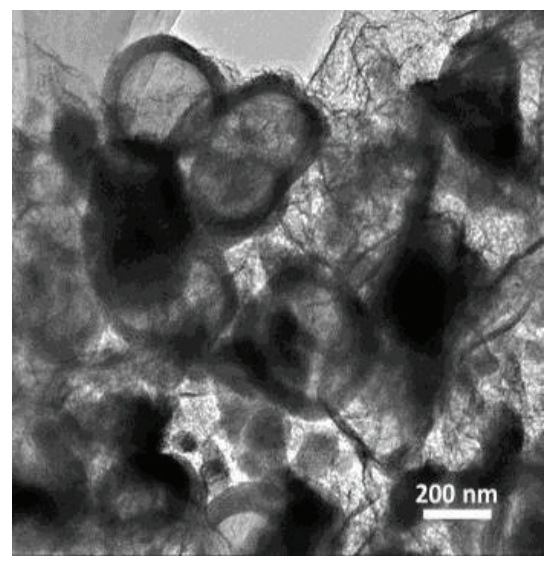

(c)

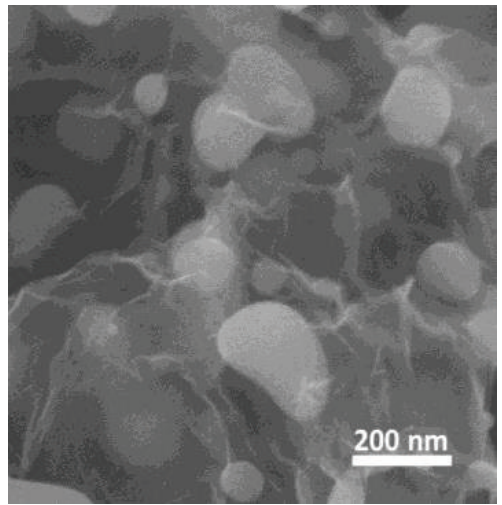

(b)

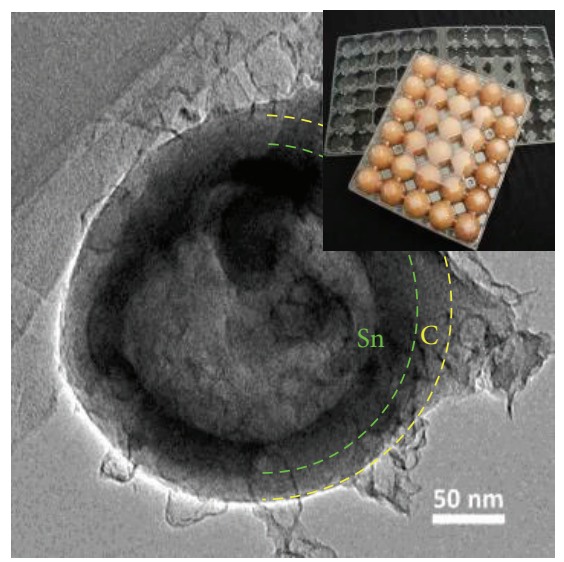

(d)

FIGURE 2: (a) XRD patterns of HSCG and crystalline Sn; (b) typical FESEM and (c and d) TEM images of HSCG. Inset of (d) shows eggs with an egg support, which is similar to HSCG nanostructure where a core-shell Sn@C is supported by planar graphene sheets.

mostly been etched off (Figure S1, ESI, available online at http://dx.doi.org/10.1155/2014/974285).

The morphologies of the HSCG were examined with field-emission scanning electron microscope (FESEM) and transmission electron microscope (TEM). Figure 2(b) clearly shows that NSs, which are with a narrow size distribution of $100 \sim 200 \mathrm{~nm}$, are uniformly anchored onto the surface of GNs. These NSs acting as the spacers prevent the restacking of GNs and stabilize the individual GNs in single or few layers, inducing the retention of open ion transportation channels. Additionally, the separated NSs guarantee the fast and sufficient contact of electrolyte with the active sites. As revealed in the SEM image, the soft two-dimensional GNs bridge the active NSs through a "plane-to-point" mode, in which a flexible conducting network is essentially constructed in electrochemical reactions. The obtained sample shows a typical IV $\mathrm{N}_{2}$ adsorption-desorption isotherm with a significant hysteresis loop, indicating that HSCG possesses a mesoporous and macroporous structure (Figure S2, ESI).
The specific surface area of HSCG is $29.51 \mathrm{~m}^{2} \mathrm{~g}^{-1}$ calculated by Brunauer-Emmett-Teller method.

The TEM images (Figures 2(c) and 2(d)) further confirm the formation of aforementioned structure. As revealed in Figure 2(d), the carbon shell with a thickness of $\sim 20 \mathrm{~nm}$ fully encapsulates the hollow Sn core to form a core-shell NS with the diameter of about $\sim 200 \mathrm{~nm}$. These NSs are tightly anchored onto the GNs even though a strong sonication treatment is used during the sample preparation for the TEM observations, suggesting a strong interaction between the NSs and GNs. Besides, the carbon shell is tightly attached to the $\mathrm{Sn}$ core, which is beneficial for mechanical reinforcement of the carbon buffer to restrict the volume expansion and enhance electronic conduction. Moreover, the void space of the hollow Sn@C is large enough to endure the volume changes during the electrochemical reaction. Such a hybrid structure, where core-shell Sn@C is supported by planar GNs, is similar to the structure of nonyolk eggs with the protection of egg support as shown in Figure 2(d) (inset). 


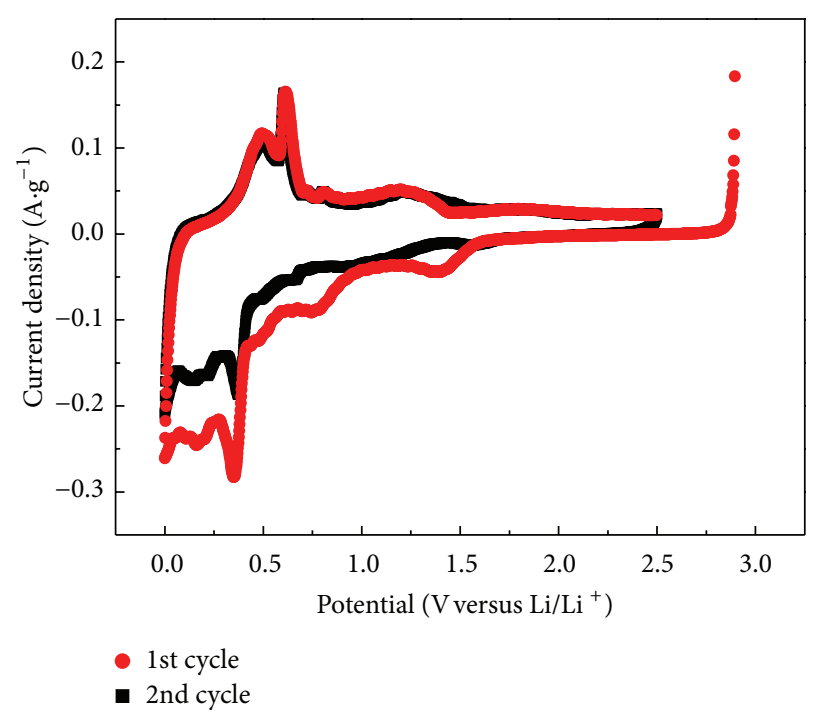

(a)

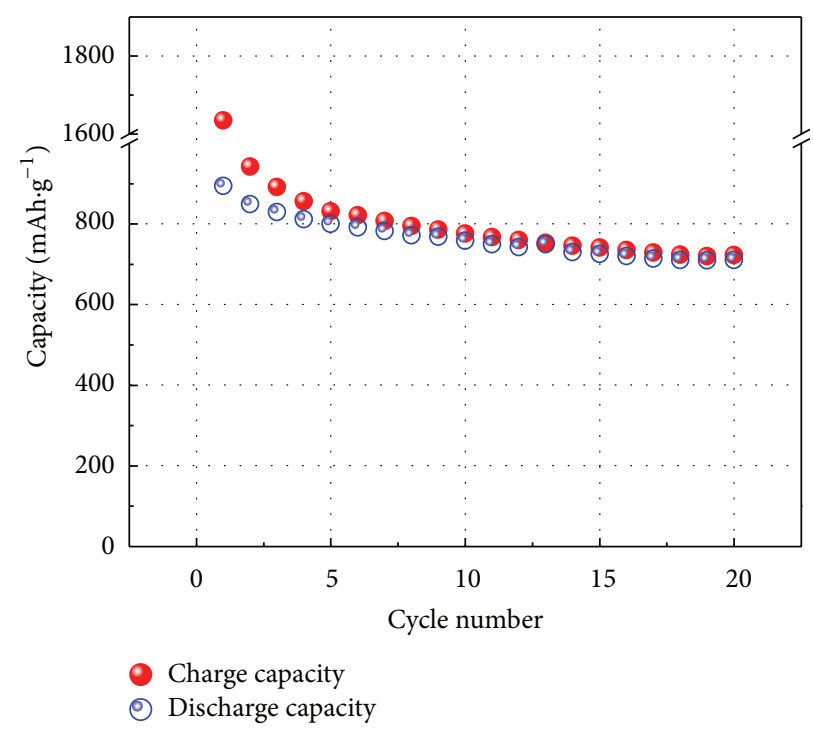

(c)

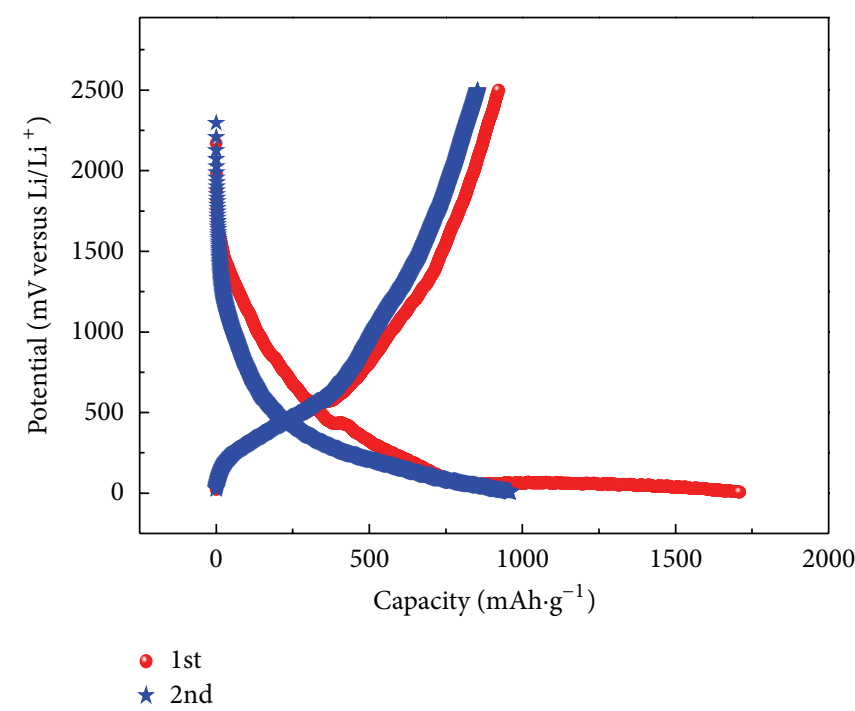

(b)

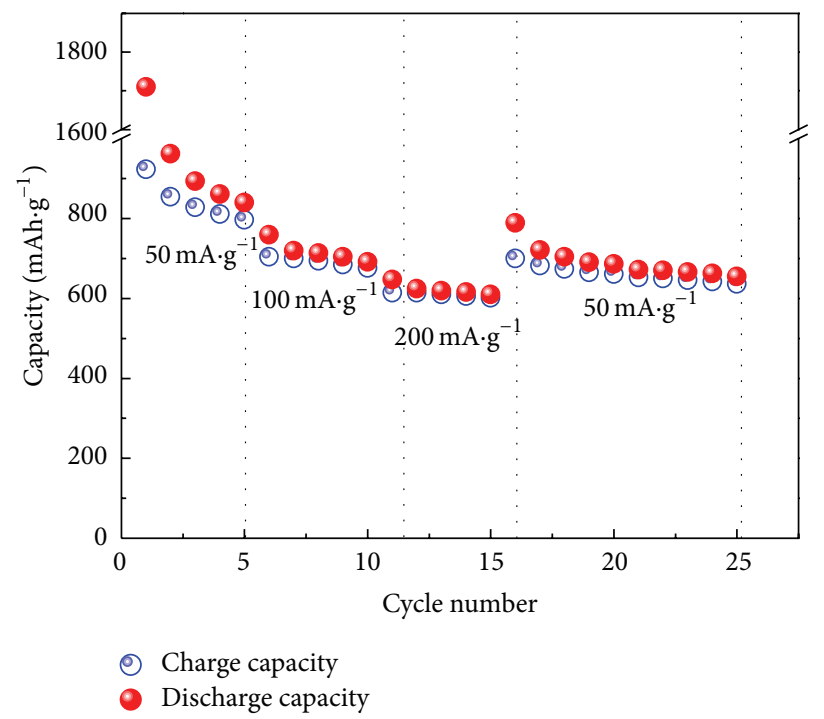

(d)

FIGURE 3: Electrochemical performance of HSCG. (a) CV profiles of the first and second cycles; (b) galvanostatic profiles at a current density of $50 \mathrm{~mA} \mathrm{~g}^{-1}$ with a cut-off potential between $5 \mathrm{mV}$ and $2.5 \mathrm{~V}$; (c) cycling profiles; (d) rate performance profile.

In order to determinate the carbon and tin fractions in HSCG, thermogravimetric analysis (TGA, Figure S3, ESI) was carried out under air atmosphere, and the result shows that HSCG contains about $70 \mathrm{wt} \%$ tin and $~ 27 \mathrm{wt} \%$ carbon.

To reveal the advantages of HSCG, its lithium insertion/extraction process is characterized with cyclic voltammetry in the range of 0.005 to $2.5 \mathrm{~V}$ at a scan rate of $0.1 \mathrm{mV} \mathrm{s}^{-1}$ [23]. The CV behavior shown in Figure 3(a) is consistent with the previous reports $[10,24]$, indicating a similar electrochemical reaction occurred. It is found that the cathodic current in the first cycle is obviously larger than that of the 2 nd cycle, especially from 1.5 to $0 \mathrm{~V}$, which is mainly due to the reduction of electrolyte on the HSCG surface to form a SEI film and the irreversible reduction of the tin oxide $\left(\mathrm{SnO}_{2}\right)$ impurity. The broad cathodic peaks at $0.3-0.7 \mathrm{~V}$ are attributed to the multistep electrochemical reduction reactions (lithiation) of $\mathrm{Sn}$ with $\mathrm{Li}$ ion to form various Li-Sn alloys. The anodic peaks at $0.4-0.8 \mathrm{~V}$ originate from the delithiation process (from the formed Li-Sn alloy phases). Based on the above discussion, it is found that the HSCG electrode shows excellent anodic reversibility.

Figures 3(b) and 3(c) show the discharge-charge voltage profiles at a current density of $50 \mathrm{~mA} \mathrm{~g}^{-1}$. The voltage profiles are consistent with that of Sn-based anodes. It is clear that the first discharge/charge capacities of the HSCG are 1710.2 and $922.7 \mathrm{mAhg}^{-1}$, respectively. The key point for such high capacity properties is derived from the synergic effect of hollow Sn@C structures with planar graphene sheets. 
The hollow Sn@C structure accommodates the volume variation and prevents pulverization of $\mathrm{Sn}$ and it also acts as the spacer to avoid face-to-face aggregation of GNs. At the same time, the graphene interlinked with each other and with the carbon shell form a continuous conducting network. The initial irreversible loss $\left(787.5 \mathrm{mAh} \mathrm{g}^{-1}\right)$ is mainly attributed to the formation of SEI film due to a relatively large interfacial surface of HSCG. At the second cycle, the HSCG anode still shows a large reversible capacity of about $853.9 \mathrm{mAh} \mathrm{g}^{-1}$, revealing a high capacity retention of $92.6 \%$ with a coulombic efficiency of $88.8 \%$. The charge/discharge property at the first two cycles is well consistent with the reactions observed from CV curves shown in Figure 3(a). After 10, 20, and 50 cycles, the reversible capacities are preserved at about $758.2,710.0$, and $659.5 \mathrm{mAhg}^{-1}$, respectively, indicating a capacity retention of $82.1 \%, 76.9 \%$, and $71.5 \%$. To the best of our knowledge, the capacities are amongst the highest values reported for Sn/C hybrid structure (see Figure S4) $[22,25,26]$. The enhanced performance of HSCG is derived from its unique infrastructure. Other than preventing the Sn NSs from aggregation, carbon shell and GNs both play the role of buffer in this hybrid to mitigate the large volume change of Sn particles associated with a conversion reaction electrode. The hollow structure of Sn also contributes to a reduction of the intrinsically large volume change.

The HSCG also shows a good rate capability and stability. As shown in Figure 3(d), at higher current densities of $100 \mathrm{~mA} \mathrm{~g}^{-1}$ and $200 \mathrm{mAg}^{-1}$, the specific discharge capacities can also reach $759.2 \mathrm{mAhg}^{-1}$ and $647.4 \mathrm{mAhg}^{-1}$. When the current density goes back to $50 \mathrm{mAg}^{-1}$, the discharge capacity returns to $788.7 \mathrm{mAh} \mathrm{g}^{-1}$, which is comparable to the initial capacity at $50 \mathrm{~mA} \mathrm{~g}^{-1}$, indicating the electrode is stable and has high reversibility. The promotion of the rate capability is attributed to the open channels and formed conductivity network mentioned in the structure discussion. Moreover, the hollow Sn shell with improved surface-to-volume ratio and reduced transport lengths for both mass and transport further improves the rate performance.

\section{Conclusion}

In summary, a novel 3D hollow Sn@carbon-graphene structure was designed and prepared by a one-pot assembly method. Such a unique hybridized nanostructure, which is similar to non-yolk eggs within a rigid egg support, is proved a highly active hollow metal@carbon structure attached on flexible and high-strength conductive GNs plane. The synergic effects, resulting from the combination of three kinds of selected infrastructures, contribute to a high reversible specific capacity of $922.7 \mathrm{mAh} \mathrm{g}^{-1}$ and excellent cyclic performance, while an outstanding rate capability is also achieved simultaneously due to the fast ion and electron transfer characteristic. This hybrid structure provides us a promising model for the design of high-performance anode material for LIBs.

\section{Conflict of Interests}

The authors declare that there is no conflict of interests regarding the publication of this paper.

\section{Acknowledgments}

The authors appreciate support from NSAF (no. U1330123), National Natural Science Foundation of China (nos. 51372167 and 51302146), Shenzhen Basic Research Project (nos. JC201104210152A and CYJ20130402145002430), and China Postdoctoral Science Foundation (2012M520012 and 2013T60111).

\section{References}

[1] S. Yang, X. Feng, S. Ivanovici, and K. Müllen, "Fabrication of graphene-encapsulated oxide nanoparticles: towards highperformance anode materials for lithium storage," Angewandte Chemie, vol. 49, no. 45, pp. 8408-8411, 2010.

[2] B. Luo, B. Wang, X. Li, Y. Jia, M. Liang, and L. Zhi, "Grapheneconfined Sn nanosheets with enhanced lithium storage capability," Advanced Materials, vol. 24, no. 26, pp. 3538-3543, 2012.

[3] C.-M. Park and K.-J. Jeon, "Porous structured SnSb/C nanocomposites for Li-ion battery anodes," Chemical Communications, vol. 47, no. 7, pp. 2122-2124, 2011.

[4] H.-C. Shin and M. L. Liu, "Three-dimensional porous copper-tin alloy electrodes for rechargeable lithium batteries," Advanced Functional Materials, vol. 15, no. 4, pp. 582-586, 2005.

[5] W. Lv, Y. Tao, W. Ni et al., "One-pot self-assembly of threedimensional graphene macroassemblies with porous core and layered shell," Journal of Materials Chemistry, vol. 21, no. 33, pp. 12352-12357, 2011.

[6] J. Liu, S. Z. Qiao, J. S. Chen, X. W. Lou, X. Xing, and G. Q. Lu, "Yolk/shell nanoparticles: new platforms for nanoreactors, drug delivery and lithium-ion batteries," Chemical Communications, vol. 47, no. 47, pp. 12578-12591, 2011.

[7] Y. Yao, M. T. McDowell, I. Ryu et al., "Interconnected silicon hollow nanospheres for lithium-ion battery anodes with long cycle life," Nano Letters, vol. 11, no. 7, pp. 2949-2954, 2011.

[8] W.-M. Zhang, J.-S. Hu, Y.-G. Guo et al., "Tin-nanoparticles encapsulated in elastic hollow carbon spheres for highperformance anode material in lithium-ion batteries," Advanced Materials, vol. 20, no. 6, pp. 1160-1165, 2008.

[9] Y.-S. Lin, J.-G. Duh, and M.-H. Hung, "Shell-by-shell synthesis and applications of carbon-coated $\mathrm{SnO}_{2}$ hollow nanospheres in lithium-ion battery," The Journal of Physical Chemistry C, vol. 114, no. 30, pp. 13136-13141, 2010.

[10] S. Liang, X. Zhu, P. Lian, W. Yang, and H. Wang, "Superior cycle performance of Sn@C/graphene nanocomposite as an anode material for lithium-ion batteries," Journal of Solid State Chemistry, vol. 184, no. 6, pp. 1400-1404, 2011.

[11] Y. Qiu, K. Yan, and S. Yang, "Ultrafine tin nanocrystallites encapsulated in mesoporous carbon nanowires: scalable synthesis and excellent electrochemical properties for rechargeable lithium ion batteries," Chemical Communications, vol. 46, no. 44, pp. 8359-8361, 2010.

[12] D. Deng and J. Y. Lee, "Reversible storage of lithium in a rambutan-like tin-carbon electrode," Angewandte Chemie, vol. 48, no. 9, pp. 1660-1663, 2009. 
[13] W. Lv, F. Sun, D.-M. Tang et al., "A sandwich structure of graphene and nickel oxide with excellent supercapacitive performance," Journal of Materials Chemistry, vol. 21, no. 25, pp. 9014-9019, 2011.

[14] Y. Wang, M. Wu, Z. Jiao, and J. Y. Lee, "Sn@CNT and Sn@C@CNT nanostructures for superior reversible lithium ion storage," Chemistry of Materials, vol. 21, no. 14, pp. 3210-3215, 2009.

[15] A. K. Geim and K. S. Novoselov, "The rise of graphene," Nature Materials, vol. 6, no. 3, pp. 183-191, 2007.

[16] W. Lv, D.-M. Tang, Y.-B. He et al., "Low-temperature exfoliated graphenes: vacuum-promoted exfoliation and electrochemical energy storage," ACS Nano, vol. 3, no. 11, pp. 3730-3736, 2009.

[17] F.-Y. Su, C. You, Y.-B. He et al., "Flexible and planar graphene conductive additives for lithium-ion batteries," Journal of Materials Chemistry, vol. 20, no. 43, pp. 9644-9650, 2010.

[18] F.-Y. Su, Y.-B. He, B. Li et al., "Could graphene construct an effective conducting network in a high-power lithium ion battery?" Nano Energy, vol. 1, no. 3, pp. 429-439, 2012.

[19] G. Zhou, D.-W. Wang, F. Li et al., "Graphene-wrapped $\mathrm{Fe}_{3} \mathrm{O}_{4}$ anode material with improved reversible capacity and cyclic stability for lithium ion batteries," Chemistry of Materials, vol. 22, no. 18, pp. 5306-5313, 2010.

[20] S. Chen, P. Chen, M. Wu, D. Pan, and Y. Wang, "Graphene supported Sn-Sb@carbon core-shell particles as a superior anode for lithium ion batteries," Electrochemistry Communications, vol. 12, no. 10, pp. 1302-1306, 2010.

[21] Z.-S. Wu, W. Ren, L. Wen et al., "Graphene anchored with $\mathrm{Co}_{3} \mathrm{O}_{4}$ nanoparticles as anode of lithium ion batteries with enhanced reversible capacity and cyclic performance," ACS Nano, vol. 4, no. 6, pp. 3187-3194, 2010.

[22] G. Wang, B. Wang, X. Wang et al., "Sn/graphene nanocomposite with 3D architecture for enhanced reversible lithium storage in lithium ion batteries," Journal of Materials Chemistry, vol. 19, no. 44, pp. 8378-8384, 2009.

[23] I. Stojković, N. Cvjetićanin, M. Mitrić, and S. Mentus, "Electrochemical properties of nanostructured $\mathrm{Li}_{1.2} \mathrm{~V}_{3} \mathrm{O}_{8}$ in aqueous $\mathrm{LiNO}_{3}$ solution," Electrochimica Acta, vol. 56, no. 18, pp. 64696473, 2011.

[24] L. Ji, Z. Tan, T. Kuykendall et al., "Multilayer nanoassembly of Sn-nanopillar arrays sandwiched between graphene layers for high-capacity lithium storage," Energy \& Environmental Science, vol. 4, no. 9, pp. 3611-3616, 2011.

[25] X. W. Lou, Y. Wang, C. Yuan, J. Y. Lee, and L. A. Archer, "Template-free synthesis of $\mathrm{SnO}_{2}$ hollow nanostructures with high lithium storage capacity," Advanced Materials, vol. 18, no. 17, pp. 2325-2329, 2006.

[26] F. Wang, G. Yao, M. Xu, M. Zhao, Z. Sun, and X. Song, "Largescale synthesis of macroporous $\mathrm{SnO}_{2}$ with/without carbon and their application as anode materials for lithium-ion batteries," Journal of Alloys and Compounds, vol. 509, no. 20, pp. 59695973, 2011. 

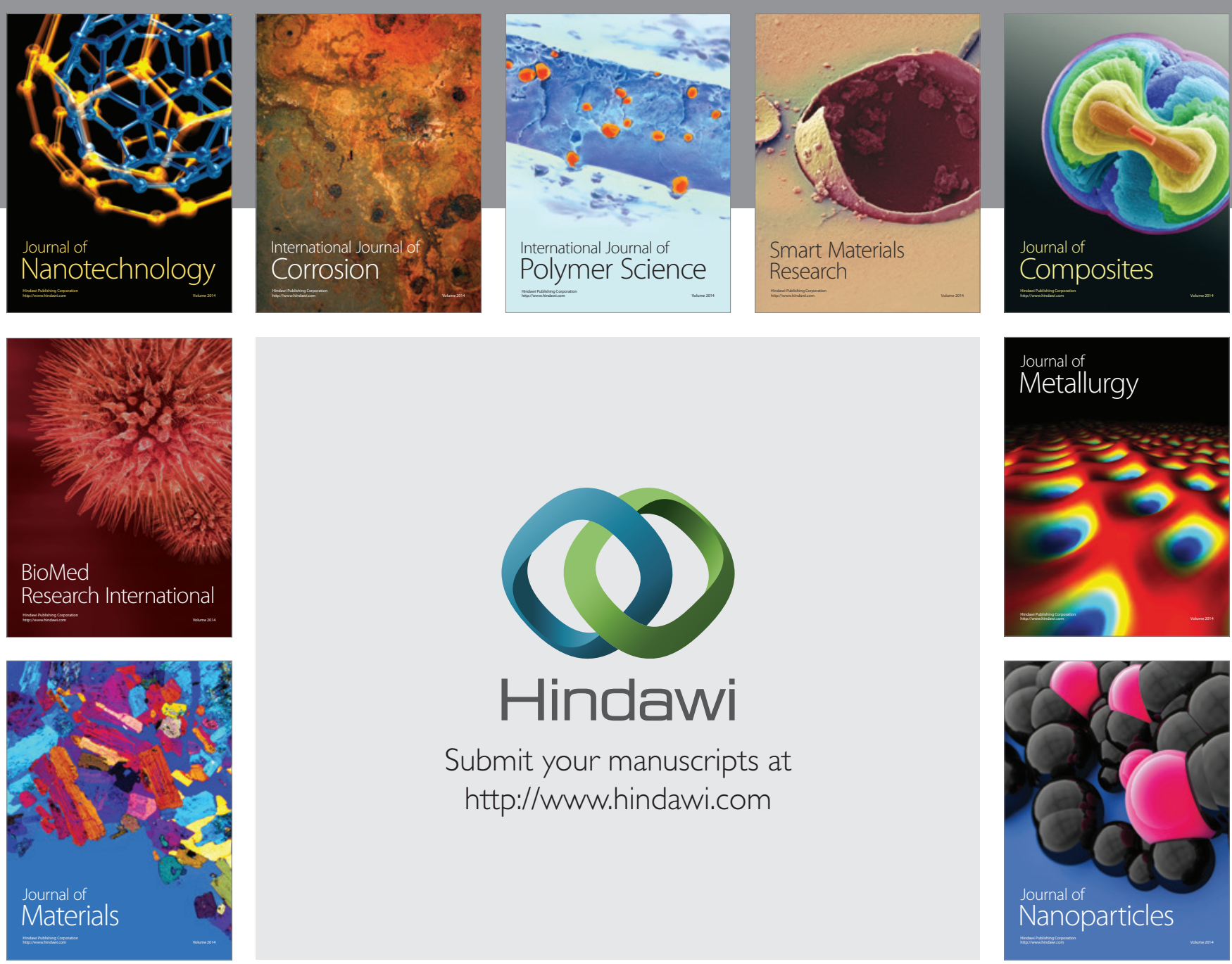

Submit your manuscripts at http://www.hindawi.com
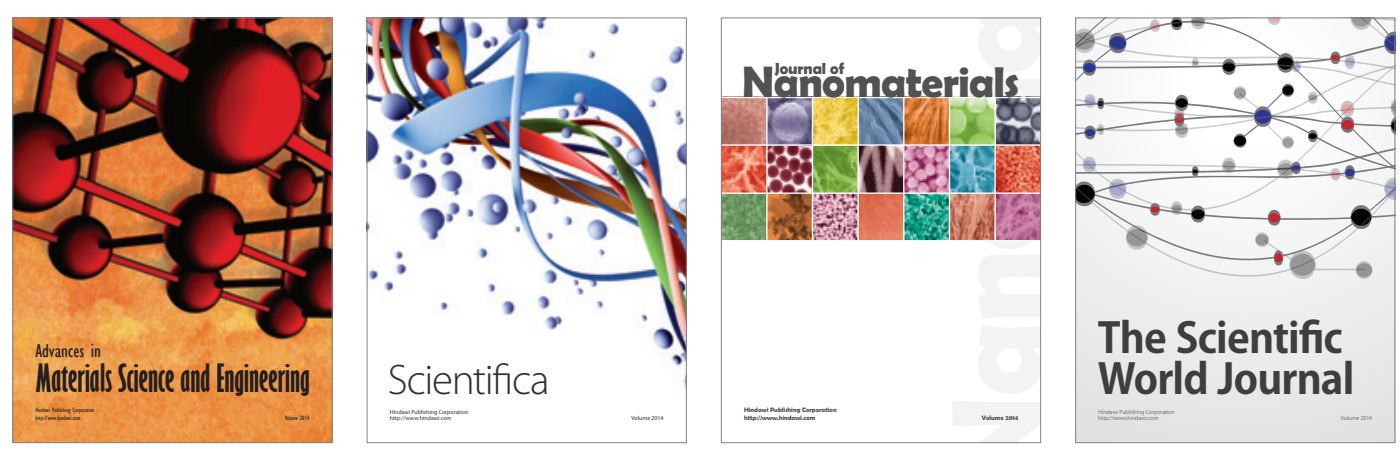

\section{The Scientific World Journal}
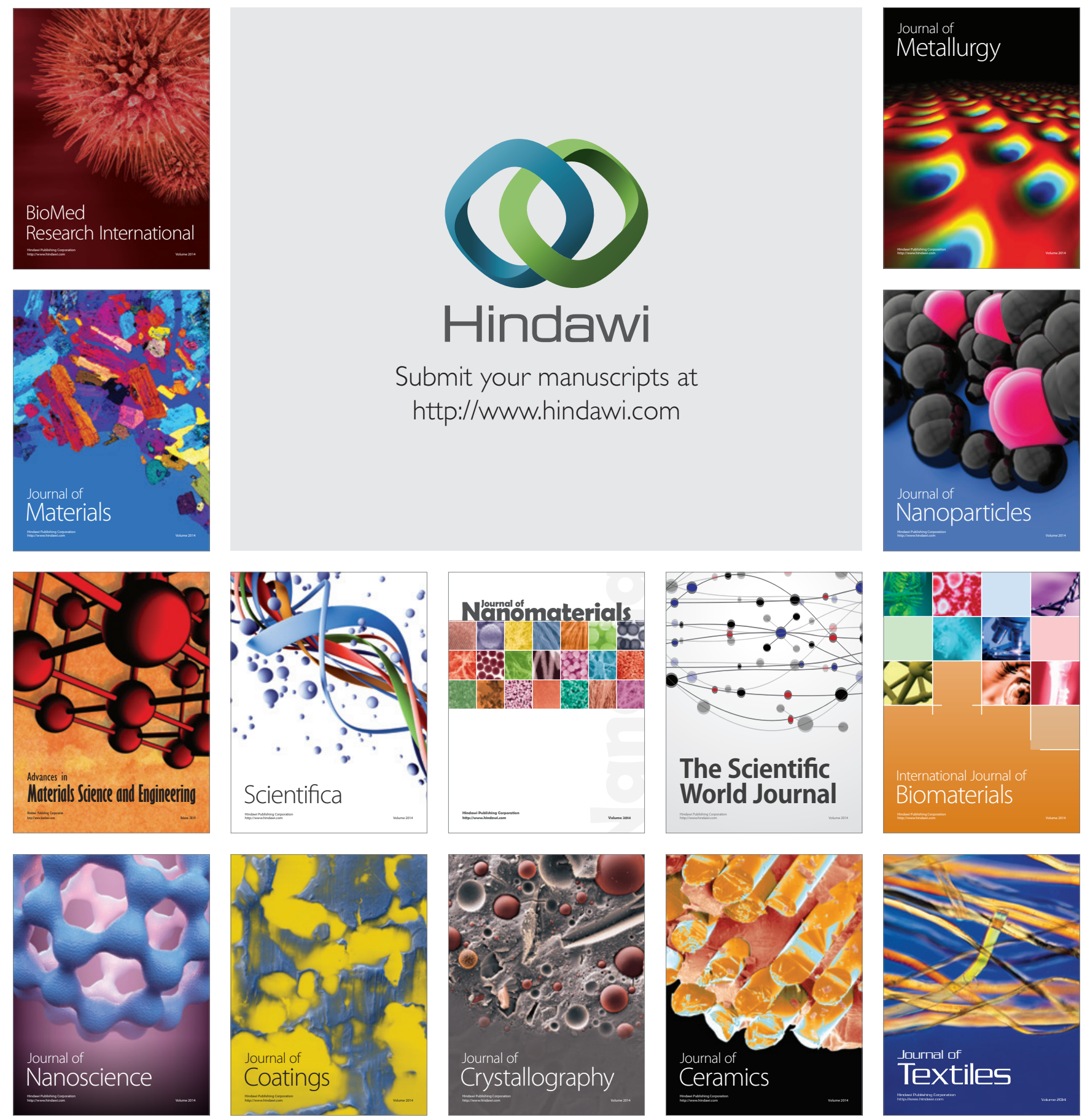